\title{
LA ECONOMÍA DE LEÓN EN EUROPA (1986-2011): SECTOR DE TECNOLOGÍA DE LA INFORMACIÓN Y LA COMUNICACIÓN EN LEÓN
}

\author{
David Abril \\ Área de Marketing \\ Departamento de Dirección y Economía de la Empresa \\ Universidad de León \\ e-mail: d.abril@unileon.es
}

1. Introducción: Las TIC y su importancia en la economía mundial y regional - 2. El sector TIC leonés: 2.1.Delimitación sector, 2.2. La evolución del sector TIC (1986-2011): 2.2.1 Primeros años, la introducción de las TIC, 2.2.2 Desarrollo de las TIC, 2.2.3 La revolución hasta nuestros días, 2.3 Sector en la actualidad, 2.4 Promoción del sector - 3. Conclusiones: Puntos importantes a tener en cuenta. Fortalezas y debilidades - Referencias

\section{Introduc ción: Las TIC y su importancia en la economía regional y mundial}

Actualmente es normal para todo el mundo el uso de todo tipo de herramientas tecnológicas como apoyo a cualquier tipo de actividad económica que realicemos. Desde los profesionales que apoyan su trabajo diario en software ofimático como hojas de cálculo o procesadores de texto, hasta los más avezados que gestionan sus empresas eficientemente gracias a la información que analizan sus software de gestión empresarial (ERP) y que reciben en tiempo real en una app en sus smartphone, todos, tienen en el sector de las Tecnologías de la Información y Comunicación (TICs) un gran aliado. Aliado que permite mejorar notablemente el funcionamiento de las empresas, haciendo todo más fácil, rápido y lo que es aún mejor, abriendo nuevas oportunidades de negocio y/o aprovechando mejor las ya existentes.

Pero, ¿cómo?

La evolución del sector TIC que analizamos en este capítulo permite ver como ha nacido $y$ crecido un sector que en 1986 apenas tenía presencia en España y que ahora tiene una importancia vital en la economía y para el resto de los sectores, en parte, como base importante para su desarrollo. Con su evolución y especialización en sus diferentes ramas de la industria se ha convertido en el más transversal de los sectores, creciendo rápidamente debido a su gran desarrollo mundial pero también gracias a la demanda existente de servicios del resto de los sectores, a los cuales les ha permitido evolucionar de manera excepcional. La automatización de procesos, la rapidez, eficiencia de ejecución y las comunicaciones cada vez más completas y eficaces han hecho que ciertos sectores hallan podido crecer empujados por la infinidad de oportunidades que ha abierto el sector TIC.

Oportunidades que aparecen debido al desarrollo del sector y que se refleja en una enorme cantidad de productos y servicios desarrollados y con aplicación directa en mejoras en procesos de empresas de otros sectores como en el mercado de usuarios particulares. 
En nuestra región se pueden ver materializadas estas oportunidades en todo tipo de sectores. Por ejemplo, en casos que ya son comunes, empresas familiares de pequeñas poblaciones rurales (50-100 habitantes), dedicadas a la fabricación de productos artesanos, en este caso de quesos, y con un mercado muy limitado hasta hace unos años pero que hoy en día se encuentran dando a conocer y vendiendo sus productos en Nueva York a través de presencia en redes sociales. Esta aplicación de comunica- ción a través de las redes sociales son servicios desarrollados en el sector TIC.

Con lo que nos encontramos ante un sector especial, aparecido en nuestra región justo al principio del periodo analizado y que ha evolucionado más que ningún otro, tanto en sus productos y servicios (Ley de Moore) como en su crecimiento como sector (evolución en facturación y número de empresas).

\section{Gráfico 1}

Ley de Moore

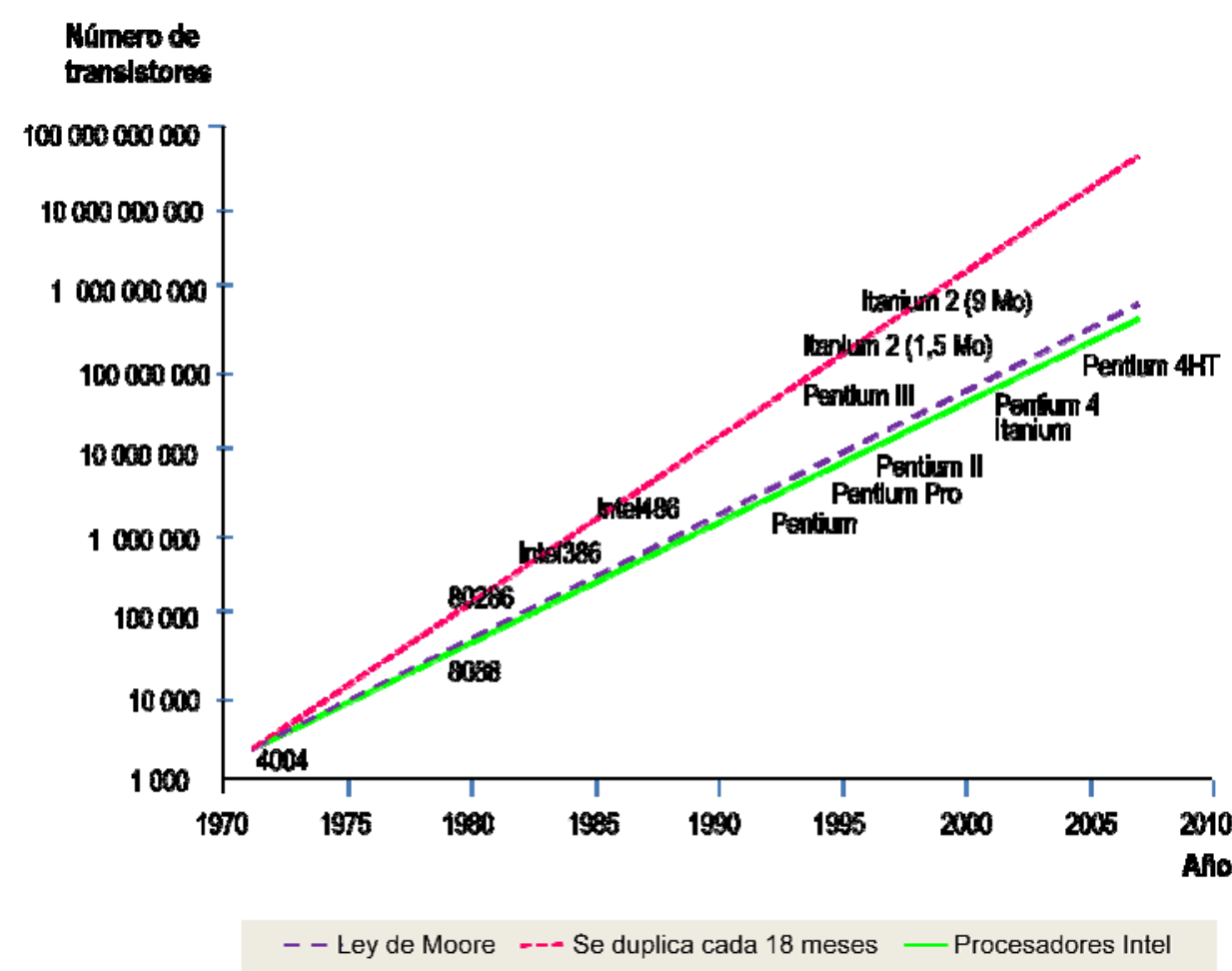

Fuente: WikimediaCommons en licencia Creative Commos por QcRef87.

La Ley de Moore demuestra como uno de los productos más necesarios dentro del sector TIC, los circuitos integrados (pieza clave en los chip), duplican cada 18 meses el número de transistores que son capaces de albergar. Como consecuencia directa nos encontramos que a la vez que los precios bajan las prestaciones suben haciendo accesible la tecnología al sector y al mercado. En 26 años se ha incrementado unas 3.200 veces el número de transistores. La ley Moore demuestra empíricamente la evolución del sector analizado.
Otras leyes como la Ley Kryder (evolución de la capacidad de almacenamiento), Ley Nielsen (evolución de la velocidad de conexión a internet) y la Ley Kaku Caveman (evolución del futuro tecnológico en desarrollos) apoyan y demuestran también la evolución de este sector evolución caracterizada por su rapidez de crecimiento y desarrollo. Todas estas leyes son el fiel reflejo de como evoluciona mundialmente las industrias TIC creando una repercusión directa en el sector regional. 
Estas premisas nos permiten hacer un análisis, comparando no solo la evolución del sector con España, Europa y el resto del mundo, sino teniendo la mejor de las guías para el análisis del sector, la propia historia de las TIC y su situación en nuestra región en cada momento.

Desde la democratización de la informática con la introducción de los ordenadores personales (más baratos y asequibles que sus predecesores y por tanto utilizables por todo tipo de empresas) hasta la internacionalización de empresas leonesas del sector con productos pioneros, desarrollados íntegramente en nuestra provincia: un análisis que recorre todo el sector y su cada vez más importante repercusión en el resto de sectores y economía.

Por último, destacar la falta de datos concisos y desagregados a nivel regional durante casi todo el periodo analizado, lo que ha llevado a su obtención a través de entrevistas a los protagonistas y a su corroboración con las dinámicas de datos más generales del mismo sector, no obstante se puede discernir la evolución regional hasta nuestros días donde si se ha podido analizar datos más explícitos.

A su vez, se ha analizado el subsector de la informática como base del sector, ya que ha sido siempre el fundamento del sector TIC y en el que se basan la mayoría de las empresas del sector desde sus comienzos.

\section{2. 日sector TIC leonés}

\subsection{Delimitac ión del sector TIC}

Dado que el sector TIC apareció en España dentro del periodo analizado, no ha tenido una clasificación legal hasta que su desarrollo nacional era notable, encontrándonos empresas regionales que han estado dadas de alta dentro de otros sectores, por ejemplo, del metal hasta la ampliación de las clasificaciones legales.

Actualmente, teniendo en cuenta los criterios de la OCDE, el sector TIC incluirá aquellas empresas en las que su actividad tiene relación con la fabricación y la prestación de servicios de las tecnologías de la información y comunicación, es decir, que sus productos y servicios tienen como objetivo recoger, procesar, transmitir y mostrar datos de manera electrónica.

Si profundizamos en cada rama del sector, podremos diferenciar:

Industrias fabricantes, cuyos productos están orientados a transmitir información, desde el procesamiento a la presentación de la misma. Incluyendo también aquellos productos que utilizan procesos para detectar, medir y/o controlar otros procesos.

Industrias de servicios, donde los productos y servicios están diseñados para trabajar con el tratamiento de la información y comunicación a través de medios electrónicos.

En el caso de España, tenemos la Clasificación Nacional de Actividades Económicas (CNAE) que se amplia gracias al análisis elaborado por AMETIC y el Ministerio de Industria, Turismo y Comercio (Cuadro 1).

Destaca los continuos debates sobre que otras actividades deben estar o no dentro del sector TIC, siendo un claro ejemplo la fabricación de cables donde dependiendo de su finalidad abre la decisión a la inclusión o no dentro del sector. Otros casos dejan ver la carencia de subcategorías que permitan una clasificación correcta. Se mantienen debates abiertos que tienen sus repercusiones en las clasificaciones, por ejemplo: la inclusión de la venta al por mayor de equipos TIC dentro de la misma categoría que la venta de equipamiento industrial, que la OCDE ha subsanado utilizando clasificaciones nacionales más detalladas que permitan un análisis correcto (CNAE 5164,5165,...) exclusivos para el sector TIC.

Por último cabe destacar la existencia de clasificaciones totalmente distintas para los diferentes organismos, encontrándonos que la Central de Bancos de España divide el sector en tres subsectores: TIC fabricación, TIC Telecomunicaciones y TIC Informática (Cuadro 1).

Aunque las diferencias de clasificaciones no son notables, sorprende la diferenciación que se hace en la última clasificación entre informática y telecomunicaciones, mientras que la OCDE agrupa todo el sector en una única clasificación. 


\section{Cuadro 1 \\ Delimitación sector}

\section{CLASIFICACIÓN DE LA OCDE}

CLASIFICACIÓN BANCO DE ESPAÑA-CENTRAL DE BALANCES

TIC Manufactura

\begin{tabular}{|c|c|c|c|}
\hline (CNAE 300) & $\begin{array}{l}\text { Fabricación de máquinas de oficina y } \\
\text { equipos informáticos }\end{array}$ & (CNAE 300) & $\begin{array}{l}\text { Fabricación de máquinas de oficina y } \\
\text { equipos informáticos }\end{array}$ \\
\hline (CNAE 313) & $\begin{array}{l}\text { Fabricación de hilos y cables eléctricos } \\
\text { aislados }\end{array}$ & (CNAE 313) & $\begin{array}{l}\text { Fabricación de hilos y cables eléctricos } \\
\text { aislados }\end{array}$ \\
\hline (CNAE 314) & $\begin{array}{l}\text { Fabricación de acumuladores y pilas } \\
\text { eléctricas }\end{array}$ & (CNAE 321) & $\begin{array}{l}\text { Fabricación de válvulas, tubos y otros } \\
\text { componentes electrónicos }\end{array}$ \\
\hline (CNAE 321) & $\begin{array}{l}\text { Fabricación de válvulas, tubos y otros } \\
\text { componentes electrónicos }\end{array}$ & (CNAE 322) & $\begin{array}{l}\text { Fabricación de transmisores de } \\
\text { radiodifusión y televisión, y de aparatos } \\
\text { para la radiotelefonía y radiotelegrafía con } \\
\text { hilos }\end{array}$ \\
\hline (CNAE 322) & $\begin{array}{l}\text { Fabricación de transmisores de } \\
\text { radiodifusión y televisión, y de aparatos } \\
\text { para la radiotelefonía y radiotelegrafía con } \\
\text { hilos }\end{array}$ & (CNAE 323) & $\begin{array}{l}\text { Fabricación de aparatos de recepción, } \\
\text { grabación y reproducción de sonido e } \\
\text { imagen }\end{array}$ \\
\hline (CNAE 323) & $\begin{array}{l}\text { Fabricación de aparatos de recepción, } \\
\text { grabación y reproducción de sonido e } \\
\text { imagen }\end{array}$ & (CNAE 332) & $\begin{array}{l}\text { Fabricación de instrumentos y aparatos de } \\
\text { medidas, verificación, control, navegación y } \\
\text { otros fines, excepto equipos de control para } \\
\text { procesos industriales }\end{array}$ \\
\hline (CNAE 332) & $\begin{array}{l}\text { Fabricación de instrumentos y aparatos de } \\
\text { medidas, verificación, control, navegación y } \\
\text { otros fines, excepto equipos de control para } \\
\text { procesos industriales }\end{array}$ & (CNAE 333) & $\begin{array}{l}\text { Fabricación de equipo de control de } \\
\text { procesos industriales }\end{array}$ \\
\hline (CNAE 333) & $\begin{array}{l}\text { Fabricación de equipo de control de } \\
\text { procesos industriales }\end{array}$ & \multicolumn{2}{|c|}{ TIC Comunicaciones } \\
\hline \multicolumn{2}{|l|}{ TIC Servicios } & (CNAE 642) & Telecomunicaciones \\
\hline (CNAE 516) & $\begin{array}{l}\text { Comercio al por mayor de maquinaria y } \\
\text { equipo }\end{array}$ & \multicolumn{2}{|c|}{ TIC Informática } \\
\hline (CNAE 642) & Telecomunicaciones & (CNAE 721) & Consulta de equipo informático \\
\hline (CNAE 713) & Alquiler de maquinaria y equipo & (CNAE 722) & $\begin{array}{l}\text { Consulta de aplicaciones informáticas y } \\
\text { suministro de programas de informática }\end{array}$ \\
\hline (CNAE 721) & Consulta de equipo informático & (CNAE 723) & Procesos de datos \\
\hline (CNAE 722) & $\begin{array}{l}\text { Consulta de aplicaciones informáticas y } \\
\text { suministro de programas de informática }\end{array}$ & (CNAE 724) & $\begin{array}{l}\text { Actividades relacionadas con bases de } \\
\text { datos }\end{array}$ \\
\hline (CNAE 723) & Procesos de datos & (CNAE 725) & $\begin{array}{l}\text { Mantenimiento y reparación de máquinas } \\
\text { de oficina, contabilidad y equipo informático }\end{array}$ \\
\hline (CNAE 724) & $\begin{array}{l}\text { Actividades relacionadas con bases de } \\
\text { datos }\end{array}$ & (CNAE 726) & $\begin{array}{l}\text { Otras actividades relacionadas con la } \\
\text { informática }\end{array}$ \\
\hline (CNAE 725) & $\begin{array}{l}\text { Mantenimiento y reparación de máquinas } \\
\text { de oficina, contabilidad y equipo informático }\end{array}$ & & \\
\hline (CNAE 726) & $\begin{array}{l}\text { Otras actividades relacionadas con la } \\
\text { informática }\end{array}$ & & \\
\hline
\end{tabular}

Fuente: El sector de las tecnologías de la información y comunicación en España en el contexto europeo. e-business Center PriceWaterhouseCoopers \& IESE/OCDE y Banco de España. 
A nivel nacional también se debe destacar la clasificación que hace AMETIC junto al Ministerio de Industria, Turismo y Comercio, mucho más especializada al ser creada desde el propio sector. El mismo sector se denomina como "Hipersector TIC" donde tienen cabida cualquier actividad económica con relación directa con la electrónica, telecomunicaciones, contenidos digitales, etc., agrupándolos en ocho grandes bloques (Cuadro 2).

\section{Cuadro 2}

Clasificación actual hipersector TIC

\begin{tabular}{|c|c|}
\hline Componentes electrónicos & $\begin{array}{l}\text { Tubos } \\
\text { Semiconductores } \\
\text { Cables } \\
\text { Componentes pasivos y electromecánicos } \\
\text { Otros componentes, pilas y antenas } \\
\text { Subcontratación electrónica }\end{array}$ \\
\hline Electrónica de consumo & $\begin{array}{l}\text { Audio } \\
\text { TVC } \\
\text { Decodificadores de TV } \\
\text { Cámaras fotográficas digitales } \\
\text { Video } \\
\text { Soportes magnéticos } \\
\end{array}$ \\
\hline Electrónica profesional & $\begin{array}{l}\text { Instrumentación y equipos didácticos } \\
\text { Electrónica de defensa } \\
\text { Electrónica industrial } \\
\text { Electromedicina } \\
\text { Radiodifusión y televisión } \\
\text { Integración e instalación }\end{array}$ \\
\hline Industrias de telecomunicación & $\begin{array}{l}\text { Equipamientos de telecomunicación } \\
\text { Integración de sistemas y servicios asociados }\end{array}$ \\
\hline $\begin{array}{l}\text { Operadores/proveedores de servicios de } \\
\text { telecomunicación }\end{array}$ & $\begin{array}{l}\text { Servicios portadores y fijos } \\
\text { Servicios móviles } \\
\text { Transmisión/conmutación de datos } \\
\text { Servicios de telecomunicación por cable } \\
\text { Servicios de acceso a Internet } \\
\text { Otros servicios }\end{array}$ \\
\hline Tecnologías de la información & $\begin{array}{l}\text { Hardware } \\
\text { Software } \\
\text { Servicios T1 }\end{array}$ \\
\hline Contenidos digitales & $\begin{array}{l}\text { Audiovisual (TV/radio) } \\
\text { Cine/Vídeo } \\
\text { Música } \\
\text { Internet } \\
\text { Publicaciones digitales, contenidos para la educación y para e-Book } \\
\text { Videojuegos } \\
\text { Contenidos para móviles }\end{array}$ \\
\hline Otras actividades TIC & $\begin{array}{l}\text { Electrónica del automóvil } \\
\text { Mantenimiento y comercio de equipos electrónicos y componentes } \\
\text { Consolas de videojuegos }\end{array}$ \\
\hline
\end{tabular}

Fuente: Mapa Hipersectorial AMETIC (abril, 2012). 
Al término del periodo analizado y desde un punto de vista económico, en España el sector facturaba anualmente (2010) 93.861 millones de euros, siendo la mayoría (46\%) de servicios de telecomunicaciones. Mientras en nuestra región y para el mismo momento (2010) el sector estaba facturando aproximadamente 147 millones de euros, teniendo una mayoría en las actividades de programación, gestión documental, servicios TIC y Comercio (14\%, $13 \%, 13 \%$ y $11 \%$, respectivamente).

\subsection{La evolución del sectorTIC (1986-2011)}

\subsubsection{Primeros años, la introduc ción de las TIC}

En el caso del sector TIC, el principio del periodo analizado coincide con el primer de los tres puntos críticos que han marcado el sector. En este caso, el nacimiento. La informática se democratizo y empezó a vislumbrar su futuro con la salida al mercado de los primeros PC (ordenadores personales) que ponían al alcance de todo el mundo el uso de ordenadores, hasta ahora reservado a grandes corporaciones debido a los excesivos precios de los microordenadores. Siendo el año 86 en el que empezaba a llegar a España los primeros PCs con discos duros asequibles, lo que permitía trabajar con cierta libertad de programación y almacenamiento de datos y abría enormes posibilidades de uso a empresas y usuarios particulares.

En León destacaba una pronta presencia de productos informáticos en las empresas, aunque hasta este momento había estado reservado a grandes organizaciones como bancos, grandes corporaciones, etc., siendo un claro ejemplo de pioneros en el uso de la informática empresarial el Diario de León entre otros.

Para dar servicio a esta nueva demanda de productos y servicios que empezaba a existir, ya había presencia desde principio de los 80 de concesionarios de empresas nacionales y multinacionales como IBM, Olivetti y la catalana Gispert, aunque aún estaban más orientadas al servicio y venta de microordenadores.
En el momento en el que el PC esta en el mercado, empresas que hasta el momento estaban dedicadas a las maquinas de escribir, fotocopiadoras y hacen su natural evolución hacia estas nuevas herramientas que aportan nuevas oportunidades a las empresas: Hispana Electrónica Castellana, Nivel Cuenta León (Concesionario de IBM), Santigosa (Ponferrada) ... Estas son las más representativas de un pequeño sector que empieza a nacer en nuestra región en esos momentos.

Si bien, destaca que aún es un sector naciente y la demanda es baja, ya que a pesar de una clara tendencia hacia la bajada de precios en los productos, aún siguen siendo precios al alcance de pocas empresas y productos limitados debido a sus características: aún no hay ningún estándar dentro del software que permita a los desarrolladores crear software común para la mayoría del mercado. Esto exige a las empresas desarrollos costosos de software a medida además del desembolso del coste de la infraestructura. Aún así el sector va emergiendo gracias a las nuevas oportunidades que abre para el resto de sectores las empresas TIC, creciendo poco a poco el sector con un aumento regular de las empresas en nuestra región.

La llegada al mercado de sistemas operativos más fáciles de usar y que estandarizan el mercado ayudan a una mayor rapidez de desarrollo del sector. Destaca la evolución de los sistemas operativos de Microsoft, que durante este periodo evolucionan del MS DOS a Windows 95, copando el mercado generalista y haciendo que cada vez sea más rápida la introducción de la informática a todo tipo de usuarios. Esto se debe principalmente a la facilidad de uso y a la estandarización de un sistema que permite el despegue de un subsector de desarrollo de software para un público más general.

Como dato representativo, se contabilizan ventas en empresas del sector de hasta 204 ordenadores personales (ventas de ordenadores personales Victor en Navidades de 1990, Hispana Electrónica Castellana S.L.) en una sola campaña de Navidad, lo que permite ver un futuro prometedor para el sector teniendo en 
cuenta que los ordenadores personales aún tenían un precio bastante alto para el momento (199.000 ptas.) y aún así la demanda era notable.

Durante este periodo el sector no deja de crecer regularmente, tanto en empresas dedicadas a la venta directa como al desarrollo de aplicaciones a medida y servicios de mantenimiento, contabilizándose en 1995 aproximadamente 15 empresas en el sector TIC en nuestra provincia (APEINTEL).

La utilización de software empresarial empieza a generalizarse en este subperiodo apoyando a las empresas con software que facilita la contabilidad, realización de nóminas, y todo tipo de gestiones y procesos.

A finales de periodo empieza a haber un cambio de tendencia, dejando de centrarse en desarrollos de software a medida para generalizarse el uso de softwares comunes desarrollados por terceros. Estos se caracterizan por ser implementados a las empresas con modificaciones a medida para cada caso, lo cual permite ventajas a las mismas en costes de software y actualizaciones, siendo un punto más a favor para el desarrollo del sector.

Por último destaca a nivel de comunidad autónoma la creación del Parque Tecnológico de Boecillo en la provincia de Valladolid. Este hecho es un movimiento estratégico de gran valor para el sector, ya que atrae a nuestra comunidad autónoma una enorme presencia de las empresas TIC más importantes del panorama nacional del momento.

\subsubsection{Desamollo de las TIC 1995-2003}

En el verano de 1995 llegaba al mercado la nueva versión del sistema operativo de Microsoft, Windows 95. Un cambio sustancial que facilitaría la expansión de la informática al ser más intuitivo, visual y con ciertas características que facilitaban el uso para las personas menos experimentadas (capacidades de plug \& play, facilidad de acceso a Internet,...). En este punto las pymes españolas y también las leonesas, empezaban a tener en la informática y las telecomunicaciones una herramienta que pasaba a ser esencial y que se iba instaurando en la mayoría de los puestos de trabajo que componía cada organización. Ya fuera a través de las herramientas de ofimática o con software de gestión (punto crítico en estos momentos de cambio de tendencia el subsector de software, de desarrollos a medida a desarrollos comunes de terceros), las TIC empezaban a tener una alta presencia y a reafirmar su utilidad y necesidad para las empresas, tendencia que se confirmaba en todos los ámbitos a través del crecimiento del sector mundial y del mercado con el número de usuarios (crecimiento general desde 1990 con 100 millones de usuarios de informática en todo el mundo a los más de 14000 millones a final del 2003).

Estos 14.000 millones de usuarios de informática mundiales, se representaban en nuestra provincia con un alto crecimiento de la demanda de servicios y productos TIC, que junto a una evolución constante de los productos y una tendencia de precios a la baja, permitía al sector un rápido crecimiento regular en tiendas de informática y empresas de servicios.

En el mercado empresarial, cada vez son más las empresas que basan su modernización en el uso de herramientas TIC, siendo acompañada esta tendencia por el crecimiento del sector descrito anteriormente y por el desarrollo mundial de las TIC. En gran parte gracias al nacimiento de Internet y al desarrollo de software que cada vez permiten más oportunidades para trabajar en todo tipo de tareas.

Destaca dentro del sector TIC en nuestra provincia la empresa LESEIN, pionera en el servicio de acceso a internet nacional a la vez que lo desarrollaban empresas nacionales como Telefónica con Infovía. Estos nuevos servicios de correo electrónico y acceso a internet ofrecidos por la empresa leonesa fueron llevados por Caja España a sus clientes, con lo que a pesar de ser un sector con poca historia y aún pocas empresas, en León ya se destacaba en el panorama nacional.

Con la estandarización que se había establecido en el anterior subperiodo, centrándose en los sistemas operativos de Microsoft, y la rápida evolución de internet, el sector no hizo más que 
prosperar durante todo este tiempo, llegando a finales de 2003 a más de 30 empresas en el sector regional y una descentralización del mercado debido a la proliferación de todo tipo de empresas dedicadas a las TIC y que abarcaban todo tipo de servicios y productos: desde telefonía móvil hasta ordenadores personales. Aunque la tendencia de venta al por menor llegaría a su fin en los siguientes años debido a la introducción en el mercado de grandes superficies con precios muy competitivos, limitando las ventas de producto de empresas pequeñas dentro del sector.

Cabe destacar que durante este subperiodo no dejan de aumentar el número de usuarios empresariales y personales de las TIC, siendo internet (correo electrónico, posibilidades de recursos y comunicación...), uno de los grandes impulsores de la tendencia y la evolución del producto y software desarrollado el otro. Todo ello sitúa al sector en un momento ideal para su siguiente evolución sobre todo debido a las, cada vez, más polivalentes soluciones y servicios que las TIC pueden dar a todo tipo de usuarios.

\subsubsection{De la revolución hasta nuestros días 2003-2011}

En este último subperiodo analizado la tendencia se hace aún más positiva y el crecimiento pasa a ser exponencial. El sector TIC sigue evolucionando gracias a las características descritas en el anterior subperiodo, pero esta evolución se hace aún más rápida y potente debido a la cantidad de oportunidades que se generan dentro de las TIC. De unos 1.400 millones de usuarios en todo el mundo, se llegan a más de 5.000 millones a final del periodo, siendo además complementado por todo tipo de productos nuevos como smartphones, tablets, etc., que ayudan a que el mercado siga creciendo y la evolución sea cada vez mayor.

\section{Gráfico 2}

\section{Evolución de usuarios de informática}

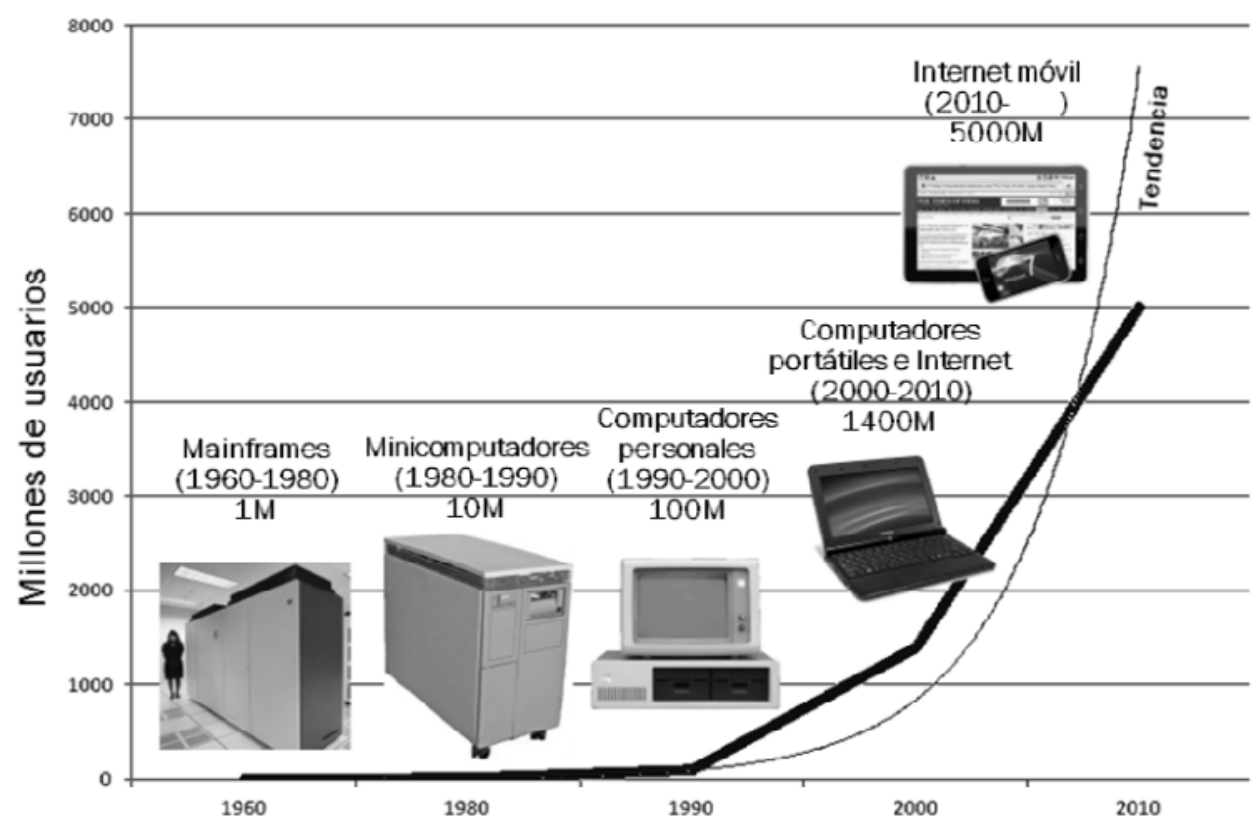

Fuente: Salmerón (2012). 
Mientras que en las TIC la evolución de productos y servicios sigue creciendo positivamente y el mercado aumenta, dentro del sector se producen cambios significativos: muchas de las empresas que habías proliferado en el subperiodo anterior, dedicadas a desarrollo de software a medida y a venta al por menor, se ven directamente afectadas por la aparición de una gran competencia con la entrada y especialización de las grandes superficies y sus precios competitivos o el desarrollo de software común que se implementa según las necesidades de los clientes.

En el caso de la venta de producto, la evolución del sector se ve marcada por la perdida de competitividad de las empresas pequeñas en la venta al por menor. Esta afecta rápidamente el mercado de usuarios particulares, aunque menos notablemente al mercado empresarial. Durante este periodo muchas empresas pequeñas se ven abocadas al cambio de sus modelos de negocio o al cambio de especialización de productos y servicios para ajustarse al mercado.

Por otro lado el sector evoluciona en otros ámbitos dado el creciente mercado y su cada vez más alta demanda de servicios, productos y software. Con la evolución de las TIC, durante este periodo se crean muchas nuevos productos y servicios que son demandados por las empresas, haciendo a finales del periodo que el sector TIC se convierta en el "Hipersector TIC" (AMETIC, Las tecnologías de la información en España, 2011) pasando de aglutinar unos pocos tipos de empresa hasta los casi 40 tipos en los que AMETIC los clasifica actualmente y que basan sus actividades en todo tipo de tecnologías de la información y comunicación.

Destaca en este subperiodo las bases que se habían establecido durante los anteriores como impulsoras del importante crecimiento del sector. De la estandarización y generalización de las TIC se pasa a un tiempo donde ya existen nativos digitales (Ferrer-Mico, 2012) que hacen de las TIC un uso continuo en todo tipo de ámbitos de su vida, potenciando al mercado y acompañando el desarrollo de oportunidades.
Durante este periodo se desarrollan todo tipo de nuevos productos, llegando a un mercado con varios estándares de sistemas operativos y productos al que el sector se adapta con notable facilidad, ofreciendo soluciones para los más generalistas y potenciando de nuevo el sector TIC con más amplitud de mercado.

Así por ejemplo nos encontramos con que hay submercados TIC que han evolucionado de manera sobresaliente como el de los smartphones y tablets, solapándose en cubrir algunas necesidades de productos más maduros pero complementándoles en gran medida. Dentro de estos productos se han creado nuevas industrias a pesar de no haber un estándar definido.

En el caso de los smartphones tenemos un predominio del mercado de Android (sistema operativo abierto de Google) y de iOS (Sistema operativo cerrado de Apple) que conviven eficientemente y dan paso a nuevas industrias y oportunidades. En el caso de nuestra región teniendo actualmente hasta cuatro empresas especializadas en el desarrollo de software (Apps) para estos sistemas que como en el resto de industrias de este sector, abre un número ilimitado de posibilidades a todo tipo de usuarios y mercado a empresas.

Por otro lado, un mercado formado por usuarios cada vez más expertos y acostumbrados a las TIC, facilita que el mercado se multiplique, y junto a la posibilidad del sector de atender todo tipo de especialización, la estandarización deja de ser necesaria para la evolución. Esto da paso a industrias TIC con diferentes estándares que en este periodo ayudan a que el mercado evolucione de manera más plural y polivalente. Un claro ejemplo es el aumento de usuarios de otras plataformas en PCs, siendo cada vez menos mayoritario el uso del sistema operativo de Microsoft, Windows (que tanta importancia tuvo en el desarrollo del sector), para dar paso a un mercado donde tienen cada vez mayor presencia otros agentes (Ubuntu/Linux, OS X/Apple,...), dando como resultado un mercado más competitivo, rico y con más oportunidades para el sector. 


\section{Gráfico 3}

Top 5 de Sistemas Operativos en España (enero-diciembre 2011)

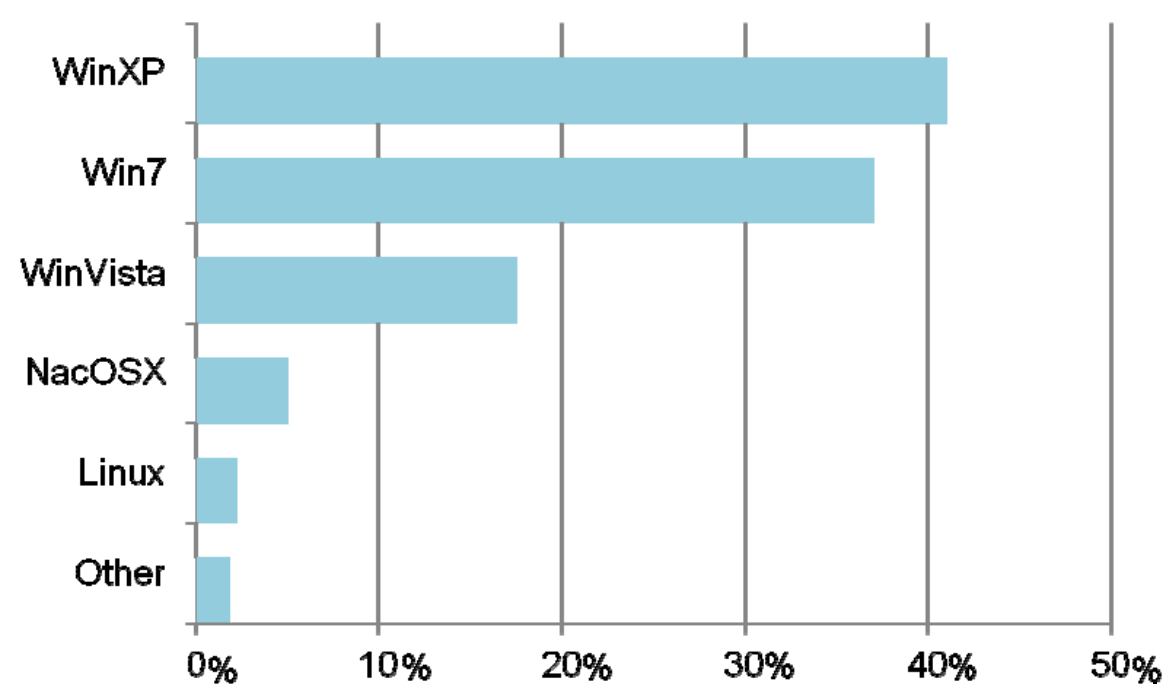

Fuente: StatCounter Global Stats. (2012).

Hasta este último periodo los sistemas operativos de Microsoft, Windows, habían sido los que claramente tenían el mercado monopolizado, pero empieza a haber cambios que una vez establecida la base, ayudan a mejorar esta industria TIC, A finales de 2011 hay una clara evolución de uso de sistemas operativos hasta ahora meramente presenciales (OS $X$, LinuX,...) que aumentan su uso en el mercado hasta niveles notables. Actualmente tanto Mac OS X (Apple) como Linux (software libre) siguen evolucionando su participación en el mercado, ayudando al crecimiento de la industria.

En esta última evolución, las comunicaciones empiezan a ser la base fundamental del sector, destaca otra de las características que hacen posible tan notable crecimiento: Internet.

Internet aporta comunicaciones y servicios que permiten expandir el mercado potencial mucho más allá de lo que se podía esperar de un sector normal. A su vez proporciona nuevas herramientas al resto de resto de sectores, creando una nueva industria que produce $y$ potencia las restantes.
En este caso, el sector también se ve potenciado por la incorporación de las TIC en muchos ámbitos de la vida produciéndose un efecto en cadena. No solo destacan aquellas empresas que usan las TIC para mejorar sus servicios y productos, motivando su uso a todo tipo de público, sino también por el aprovechamiento que hacen de ellas los organismos públicos para mejorar la comunicación y atención a la población. Todo ello se converge en un impulso de todas las industrias TIC.

Un claro ejemplo se ve por parte de la Agencia Tributaria. La Agencia Tributaria actualmente obliga a que todo tipo de empresa tenga un correo electrónico para poder recibir las notificaciones telemáticas. Por otro lado ayuntamientos y otros organismos han adoptado las TIC para facilitar los trámites de manera notable y su vez mejorando el servicio para ambas partes.

Todo esta evolución se ve reflejada en el ya sector TIC de nuestra provincia, que a pesar de los diferentes cambios de modelos de negocio que exigen estas industrias tan dinámicas, las empresas leonesas son capaces de adaptarse y aprovechar llegando en el 2010 a unas 263 
empresas contabilizadas en el sector (ALETIC, 2010). Un notable crecimiento en este último subperiodo que es reflejo de la evolución de las TIC como hemos podido ver anteriormente y que se caracteriza principalmente no solo por la evolución de las industrias ya existentes sino por la creación de nuevas industrias y el potenciador que ejerce el uso de estas tecnologías por parte del público en general y de manera más mayoritaria. La aparición y desarrollo de internet y la adopción por parte de todo tipo de agentes complementa estas características.

Todo esto permite que muchas empresas localizadas dentro del sector TIC leonés estén trabajando fuera de la región e incluso internacionalmente aportando sus servicios y productos de manera global desde León.

Por último cabe destacar la relevancia del sector a todos los niveles. Dentro de este subperiodo todos los agentes son conscientes de su importancia como sector emergente, pero a su vez de la relevancia que tiene para el desarrollo del resto de sectores, de su peso en la economía y del desarrollo del país.

En España el sector es potenciado de diferentes maneras a través de varios organismos y de la administración (ejemplo principal Red.es, la entidad pública del Ministerio de Industria, Energía y Turismo, MINETUR, encargada de consolidar el desarrollo de la Sociedad de la Información en España). Por otro lado, en el caso de nuestra región, la administración lo potencia con diferentes acciones a través de todo el subperiodo, destacando la creación del Parque Tecnológico de León, el cual es inaugurado en 2008 y que se ha consolidado como espacio de reunión de las principales empresas del sector TIC en nuestra provincia. Este no sólo ha sido un espacio para la evolución del sector regional sino que además ha atraído empresas internacionales como Hewlett Packard que actualmente trabaja y da servicio internacional desde sus instalaciones situadas en León.

\subsection{E sector TIC en la actualidad}

El sector TIC leonés ha crecido de manera sobresaliente durante el periodo analizado, siendo un fiel reflejo de la evolución del sector TIC en todo el mundo.

A finales del periodo estudiado se contabilizan casi 300 empresas dentro del sector TIC de nuestra provincia. Teniendo datos exactos gracias al informe elaborado en 2010 por una de las dos asociaciones sectoriales que existente en la provincia, ALETIC.

\section{Localización}

En el 2009 existen 285 empresas registradas dentro del sector TIC en León. Una catalogación por zonas de situación, en vez de por localidades nos proporciona conclusiones relevantes: En la provincia de León las empresas del sector TIC se distribuye principalmente en la ciudad de León y su alfoz, estando las restantes en el Bierzo o en el resto de la provincia.

Por tanto, actualmente, casi un $70 \%$ de las empresas TIC de la región de León se encuentran concentradas en la zona de la capital. Estos datos se corresponden con los datos provinciales del PIB, per.

Tabla 1

Distribución de empresas del sector en la provincia de León

\begin{tabular}{|c|c|c|}
\hline Localidad & Número & $\%$ \\
\hline León & 156 & 54,7 \\
\hline Alfoz & 41 & 14,4 \\
\hline Bierzo & 64 & 22,5 \\
\hline Resto provincia & 24 & 8,4 \\
\hline TOTAL & 285 & 100 \\
\hline
\end{tabular}

Fuente: Informe Sectorial ALETIC (2010). 


\section{Fac turación y tamaño de empresas del sector}

El tamaño del sector TIC se establece en una estimación de 147 millones de euros en el 2009 (ALETIC, 2010), donde apenas un 10\% del total facturan más de un millón de euros anualmente. La mayoría de las empresas (60\%) facturan menos de 250.000 euros. Lo que deja ver un ecosistema de pequeñas empresas que se abren paso dentro de las diferentes posibilidades que ofrece el sector.
Los datos de tamaño de las empresas según su número de empleados siguen la misma dinámica que la clasificación por facturación, encontrándonos que casi un $88 \%$ tienen menos de 10 empleados, predominando con un $45 \%$ las microempresas de 1 y 2 empleados. Con los datos del estudio se estima que en el 2009 en el sector TIC trabajaban 2.856 personas en la región de León, existiendo una reducción interanual que bien esta relacionada con la coyuntura económica actual.

\section{Tabla 2}

Distribución de empleados en empresas del Sector TIC Leones

\begin{tabular}{|c|c|c|}
\hline Empleados & Número & $\%$ \\
\hline $1-2$ & 87 & 44,6 \\
\hline $3-4$ & 51 & 26,2 \\
\hline $5-9$ & 31 & 15,9 \\
\hline $10-19$ & 15 & 7,7 \\
\hline 20 ó & 11 & 5,6 \\
\hline TOTAL & 195 & 100 \\
\hline
\end{tabular}

Fuente: Informe Sectorial ALETIC (2010).

Dado los datos analizados para la etapa final del periodo a estudio, podemos concluir que el sector TIC se ha desarrollado eficientemente y sigue su expansión, aunque marcada por la situación económica del entorno. La facturación es muy relevante para un sector que ha ido emergiendo y el tamaño de las empresas así como el número de empleados son una imagen realista de la potencia del sector. Estamos ante un sector maduro.

\subsection{La promoción del sector}

El sector TIC en León se refuerza por diferentes agentes que han ido apareciendo en el último subperiodo y que refuerzan y empujan el desarrollo del sector en León.

\section{Asociaciones empresariales}

Destaca que en una provincia como León existan dos asociaciones empresariales para el mismo sector: APEINTEL y ALETIC. Lo que cobra coherencia cuando se analiza el tejido empresarial leonés y se tiene en cuenta que también hay dos asociaciones empresariales generales: FELE y CEL.

Ambas asociaciones TIC trabajan por el fortalecimiento del sector, canalizando los esfuerzos individuales de las empresas del sector para mejorar la generación de negocio y prestar apoyo al resto de sectores: colaborando por el bien común de toda la industria leonesa.

Destaca fuera de la región, la alta presencia de empresas leonesas en la dirección de asociaciones y patronales a nivel de comunidad autónoma (AMETIC Castilla y León y AETICAL), dejando claro el compromiso y potencial de las empresas TIC de León con el sector no solo con el sector regional sino también con el nacional.

\section{AE Seguridad}

El máximo exponente en promoción del sector TIC desde el asociacionismo esta representado en nuestra provincia por la AEI Seguridad. Esta agrupación empresarial Innovadora surge del 
polo tic generado por el INTECO y aglutina todo tipo de agentes relacionados con el sector TIC, siendo la mayoría de ellos de la provincia de León.

La AEI Seguridad de las Redes y Sistemas de Información (nombre completo, aunque conocida públicamente como AEI Seguridad) tiene como objeto el dotar de una estructura operativa al sector y en particular a las empresas que se vinculan a la agrupación para el desarrollo de un Polo Empresarial y Tecnológico. Un claro síntoma más del gran potencial y desarrollo del sector TIC en la región leonesa.

\section{Universidad de León}

La Universidad de León cobra un protagonismo esencial en el desarrollo del sector TIC en León gracias a su oferta formativa que abarca los conocimientos principalmente demandados por las empresas TIC. Destaca por ser el principal proveedor de personal técnico cualificado, siendo la mayoría de los empleos del sector leonés cubiertos por personal formado en ella.

La oferta formativa abarca desde grados hasta programas de doctorado, incluyendo grupos de $\mathrm{I}+\mathrm{D}+\mathrm{I}$ con un alto porcentaje de investigadores doctorados.

Además la Universidad de León destaca por su grado alto de involucración con el sector, participando en todo tipo de proyectos y acciones nacidas en el propio sector.

\section{Fundación Centro Supercomputac ión de Castilla y León}

FCSC es una entidad pública creada en el 2008 desde la Junta de Castilla y León y la Universidad de León con el fin de promover acciones y proyectos en los que las TIC tienen un papel principal además de aportar mediante TICs al desarrollo y mejora de competitividad de todo tipo de empresas.

Claramente relacionado con el sector TIC, el FCSC es un claro impulsor de toda la industria y su situación en León facilita la relación con las empresas leonesas.
Entre sus recursos destaca Caléndula, siendo el segundo superordenador más potente de España y estando sobre el número 180 del ranking mundial. Superordenador que destina su tiempo a partes iguales a proyectos universitarios y de empresas privadas.

\section{INTECO}

Fundado en el 2006, INTENCO es el Instituto de Tecnologías de la Comunicación, un organismo dependiente de Red.es y por tanto del Ministerio de Industria, Energía y Turismo. Con su sede oficial establecida en León y como objetivo el desarrollo de la Sociedad de la Información a través de la innovación, el desarrollo de proyectos TIC y la seguridad nacional de la información ha sido un buen catalizador para el sector TIC leonés.

Destacan entre sus acciones, el liderazgo de la AEI Seguridad, desarrollo de proyectos en colaboración con empresas leonesas y un compromiso general con todo el sector TIC.

\section{Conc lusiones: Puntos importantes a tener en c uenta. Fortalezas y debilidades}

Con el análisis del sector TIC leonés durante el periodo de 1986 a 2011 y su comparación con el desarrollo de las TIC a nivel mundial se puede concluir que las empresas leonesas han sabido aprovechar las oportunidades de este sector emergente, evolucionando y creando en este periodo un sector básico para el apoyo y desarrollo del resto de sectores y de la economía regional.

Durante el análisis del desarrollo del sector se ha visto como siempre ha sido un crecimiento regular y controlado, acompañando a la demanda y a las oportunidades generadas por el mismo. Esto ha permitido ir creando un sector maduro que ahora mismo puede ser competitivo no solo en la provincia sino a nivel nacional e internacional como demuestras ciertas empresas leonesas. Así mismo la unión del sector y agentes como la Universidad de León entre otros han producido un entorno 
autónomo que atrae empresas y genera un polo TIC significativo a nivel nacional.

Con los datos actuales se corrobora que a durante estos años se ha creado un tejido empresarial TIC fuerte que será pilar para un crecimiento continuado en los próximos años, no solo gracias a la evolución de las tecnologías que podemos comprobar a diario, sino porque la base de empresas, tamaño y crecimiento es lo suficientemente significativo para llevarlo a cabo.

Destaca también la demanda constante de productos y servicios que necesitan actualmente el resto de sectores del sector analizado, así como el compromiso de la administración y agentes de peso con el sector.

Otro punto importante que deja patente el desarrollo del sector es el alto grado de asociacionismo, así como de proyectos comunes. El compromiso de las empresas TIC de la provincia de León no solo se centra para con nuestra región, sino que hay una alta presencia y compromiso de las mismas en la dirección de asociaciones autonómicas y nacionales en el sector.

Por el contrario, el sector en León se ha visto afectado varias veces por los cambios en la evolución del mercado TIC produciendo la desaparición de parte del tejido empresarial del sector, aunque siempre se ha visto renovado con las nuevas oportunidades surgidas. Es marcado el cambio de dinámica del sector cuando entran las grandes superficies en la venta al por menos de productos informáticos, afectando gravemente a tiendas pequeñas de producto y servicio al por menor, pero contrasta con la aparición de otras pequeñas empresas dedicadas a la especialización dentro de alguna de las industrias del sector, como es el caso, por ejemplo, de la reciente aparición de varias empresas dedicadas a la creación de APPs para smartphones y tablets. Con lo cual, aunque el sector en sensible a los cambios de modelos de negocios y a la fuerza de competidores grandes que entran en juego, el sector TIC se caracteriza por su rápida evolución y la capacidad que deriva de ello para generar nuevas oportunidades de negocio a las empresas del sector.

Por último y como el resto de sectores, desde el 2008, las empresas TIC leonesas se ven claramente afectadas por la coyuntura económica actual, aunque se mantienen y las percepciones de futuro para el sector son buenas tanto por la transversabilidad del sector, como por la demanda creciente y el continuo y rápido desarrollo de las TIC a nivel mundial.

\section{Referencias}

ALETIC (2010). Estudio del sector TIC en León.

Entrevista FELE (Federación de Empresarios Leoneses) y APEINTEL (Asociación Provincial de Empresarios de Informática y Nuevas Tecnologías de León) Febrero 2013.

Ferrer-Mico, T. (2012). Nativos digitales. Journal of Feelsynapsis, 52-56.

Nota de prensa Agencia Tributaria sobre Comunicaciones y notificaciones electrónicas de la AEAT 2011.

PWC \& IESE (2009). El sector de las TIC en España en el contexto europeo: Evolución y tendencias.

Rodríguez López, I. (2008). Las tecnologías de la información y las comunicaciones en la empresa española.

Salmerón, A. (2012). Evolución de la informática.

Salmerón, A. (2013). Microsoft. La historia de Windows. 